\title{
Nasal Powder in Single-Dose Container
}

National Cancer Institute

\section{Source}

National Cancer Institute. Nasal Powder in Single-Dose Container. NCI Thesaurus. Code C162315.

Medicinal product consisting of a nasal powder presented in a single-dose container, usually with an integral device to facilitate nasal administration. 